\title{
Determinantes sociales y conductuales en salud nutricional: evidencia para Chile
}

\author{
Social and behavioral determinants on \\ nutritional health: evidence for Chile
}

\begin{abstract}
Due to the nutritional transition in the last decades, the risk factors linked to food nutrition are in the spotlight of public policy and reduction of their prevalence is a key goal in the public health promotion strategies involving food security and nutrition (FSN). Understanding the factors underlying poor nutrition status is a must in order to execute successful policy interventions in the general population. This paper analyses the impact that social and individual behavior variables have on the risk factors linked to poor nutrition (obesity, high levels of cholesterol, glycaemia and sodium) using data from the National Health Survey 2009-2010 using a Seemingly Unrelated Equations (SUR) approach in order to have a consistent estimation outcome. Findings suggest that variables linked to social environment and individual behavior have a significant impact on the food-related health risk factors, taking account for social, demographic, genetic and economic controls. Unsurprisingly, when people underestimate their nutritional status, it conduces to a greater health risk, explaining up to $6 \mathrm{cms}$ of abdominal girth and 3 points of the BMI index. Also, an insecure neighborhood and weak social networks explain part of the health risk. These results are a starting point to discuss the design of public policy regarding health and nutrition in order to promote the food security especially regarding information and education programs, where there is the possibility to strengthen the social support networks.
\end{abstract}

Key words: health risk, nutritional status, food security and nutrition, Chile, poor nutrition.

\section{INTRODUCCIÓN}

Los factores de riesgo a la salud que se relacionan con hábitos alimentarios han aumentado su prevalencia en la gran mayoría de los países desarrollados, particularmente en las últimas décadas y con una mayor incidencia en niños $(1,2)$. Las enfermedades no-transmisibles con mayor tasa de mortalidad, como las coronariopatías, hipertensión, cáncer, arritmia y diabetes están asociadas con la malnutrición, sobrepeso e inactividad física $(1,3)$. En Chile, la obesidad y sobrepeso afecta a dos tercios de la población adulta, dos quintos de los jóvenes a nivel de octavo básico y más de un $10 \%$ de los niños menores de cinco años (4).

En el contexto de la transición nutricional experimentada en las últimas décadas en Chile (y el mundo entero), se observa un incremento en la prevalencia de factores de riesgo a la salud en la población, particularmente aquellos asociados a obesidad y sedentarismo, pero también a un mayor consumo
Juan Carlos Caro S.

Facultad de Economía y Empresa, Universidad Diego Portales, Santiago, Chile.

Dirigir la correspondencia a: Sr. Juan Carlos Caro S. Facultad de Economía y Empresa Universidad Diego Portales Av. Santa Clara 797, Huechuraba, Santiago, Chile. Teléfono: 223763329 Email: juan.caro@mail.udp.cl

Este trabajo fue recibido el 30 de Septiembre de 2014 y aceptado para ser publicado el 10 de Enero de 2015.

de alimentos altos en sodio, azúcares y grasas $(5,6)$.

Cambios tanto en los contenidos como en la variedad de la oferta alimentaria disponible hacia los hogares, así como en los estilos de vida (tiempo dedicado a la alimentación y actividad física), han contribuido de manera significativa al incremento en la malnutrición, ya sea por exceso de ingesta calórica o por una dieta inadecuada en nutrientes esenciales (2). Controlando por las diferencias en la oferta de alimentos y factores socioeconómicos, existe evidencia que factores de comportamiento (conductuales) así como del entorno de los hogares (ambiente social) son relevantes en términos de su conducta en cuanto a la ingesta de alimentos y actividad física (7-10). En Chile no existen a la fecha estudios similares que analicen el impacto de estos factores en la malnutrición de la población desde una perspectiva sistémica.

El objetivo de este estudio fue analizar la relación entre los determinantes sociales y conductuales de la población 
chilena y los factores distales de riesgo a la salud vinculada a malnutrición (particularmente por exceso), a partir de los datos de la Encuesta Nacional de Salud 2009-2010. Los resultados son relevantes para la discusión actual de política pública, en particular en cuanto a que elementos considerar en el diseño de programas de información y educación alimentaria hacia los consumidores, así como proveer mecanismos que fortalezcan las redes de apoyo a nivel individual, a fin de asegurar un acceso y una apropiada utilización de la alimentación de manera que se reduzcan los factores de riesgo de salud por malnutrición.

\section{SUJETOS Y MÉTODOS Diseño}

Para este análisis se siguió un modelo teórico causal basado en Margozzini (11), donde existe un set observable de variables individuales que conduce a una mayor prevalencia de factores distales y proximales de riesgo asociados a una mala alimentación (IMC elevado, obesidad abdominal alta, colesterol alto, entre otros), los cuales a su vez elevan riesgo de presentar una enfermedad. Siguiendo el modelo, las variables individuales tienen relación con factores genéticos, sociales, conductuales y ambientales. Finalmente, el riesgo de presentar una enfermedad puede observarse indirectamente de forma consistente a través la medición del riesgo de enfermedad cardiovascular $(12,13)$. Para facilitar el análisis, podemos expresar matemáticamente la relación anterior:

$$
\begin{aligned}
& R C V_{i}=1\left(X_{i}^{\prime} \beta+W i^{\prime} \theta+\varepsilon_{i}>0\right) \\
& X_{i j}=Z_{i}^{\prime} Y+e_{i} \forall_{j}
\end{aligned}
$$

Donde la prevalencia de riesgo cardiovascular alto para el individuo (RCVi) se modela con una función indicador que está asociada a los factores de riesgo asociados a alimentación $\left(X_{i}\right)$ y otros factores $\left(W_{i}\right)$, que pueden ser de orden genético o ambiental. En este modelo, $(\beta, \theta)$ son parámetros que informan sobre el efecto que un factor de riesgo tiene sobre la posibilidad de observar un individuo con riesgo cardiovascular alto.

A su vez, cada factor de riesgo observado en el vector $X\left(x_{i j} \in X_{i}\right)$ representa el factor de riesgo nutricional (j) para el individuo (i), que puede ser modelado de forma lineal a través de un grupo de variables individuales ( $\mathrm{Zi}$ ), que pueden ser conductuales, psicosociales, ambientales o genéticas. Entonces, es el vector de parámetros que informa sobre efecto directo de una variable individual sobre el factor de riesgo, como el IMC, por ejemplo.

Tanto $\epsilon_{i}$ como $e_{i}$ son términos de error vinculados a cada una de las ecuaciones respectivas. Es importante notar que pueden existir variables incluidas simultáneamente en ( $\mathrm{Zi}$ ) y (Wi), ya que, por ejemplo, la prevalencia de RCV puede estar explicada de forma directa e indirecta por factores individuales (11).

\section{Encuesta}

La Encuesta Nacional de Salud (ENS) es una iniciativa del Ministerio de Salud de Chile, y ha sido efectuada en dos ocasiones, el año 2003, y luego entre los años 2009-2010 (14). El objetivo es obtener información poblacional sobre la prevalencia de enfermedades no transmisibles y los factores de riesgo a la salud asociados a las mismas. La encuesta es aplicada a mayores de 15 años y tiene representatividad regional y a nivel de zonas (urbano y rural). La ENS cuenta con un análisis de patrones de consumo alimentario, sedentarismo, consumo de drogas, variables psicosociales, entre otras. El número de observaciones totales varía de acuerdo a las variables consideradas ya que la encuesta se aplicó en base a dos cuestionarios separados (entrevistador y profesional médico), lo cual afecta el número de observaciones.

\section{Variables}

Para el riesgo cardiovascular, se considera un valor de 1 para aquellos tienen un riesgo alto y 0 para el resto de la población. Los factores de riesgo asociados a la alimentación considerados para este estudio fueron el estado nutricional, medido tanto por el índice de masa corporal, IMC $\left(\mathrm{kg} / \mathrm{mt}^{2}\right)$, como por la circunferencia abdominal $(\mathrm{cm})$, la ingesta de sodio (gl/día), el colesterol total ( $\mathrm{mg} / \mathrm{dL}$ ) y la glicemia total $(\mathrm{mg} / \mathrm{dL})$. La ingesta de sodio se usa como una medida para aproximar el balance de sodio-potasio, debido a dificultades de medición de dicha variable.

En cuanto a las características individuales utilizadas a partir de la encuesta, existen mediciones socioeconómicas, demográficas, ambientales (percepción del entorno), psicosociales, de dieta alimentaria, consumo de alcohol y tabaco, y actividad física. En particular, dentro de las variables ambientales, se utilizaron cuatro medidas de auto percepción de la red de apoyo individual, basadas a la respuesta sobre el grado de acuerdo/desacuerdo (con niveles decrecientes de 1 a 5) acerca de las siguientes afirmaciones: 1) a nadie le importa mucho lo que me pase, 2) es más seguro no confiar en nadie, 3) tiene alguien a quien pedir ayuda/consejo, y 4) este es una villa/barrio donde los vecinos se preocupan unos por otros ${ }^{1}$. Las demás variables ambientales, que también se miden en grado de acuerdo/desacuerdo, incluyen el acceso a apoyo económico por un cercano (medido en escala de 1 a 7) y percepción de seguridad en el barrio (escala de 1 a 5). En cuanto a los aspectos psicosociales, se utiliza la autopercepción de bienestar general y de salud (medidas en grado de acuerdo/desacuerdo en escala de 1 a 5), y una variable definida como hostilidad, la cual representa el grado en que la persona manifiesta no participar en actividades en su grupo social de referencia (estas variables vienen ya creadas y definidas en la ENS 2009-2010, todas en escala de 1 a 5).

Adicionalmente, dado que se cuenta con un autorreporte del estado nutricional, se definió una variable llamada diferencia en percepción física, medida como la diferencia entre lo autorreportado y observado, como una medida de la subestimación (+) o sobreestimación (-) sobre el estado nutricional individual. Esta variable resulta muy relevante a nivel teórico, ya que refleja el grado en que el individuo identifica correctamente su situación nutricional.

\section{Análisis estadístico}

La estadística descriptiva muestra la media y desviación estándar de las variables utilizadas en el estudio, así como la correlación simple entre los factores de riesgo y el RCV (muestra completa). Adicionalmente se estimó una regresión logística sobre el RCV usando los factores de riesgo y otras variables individuales (ecuación 1), para validar el modelo teórico. Posteriormente, el modelo estadístico para explicar los factores de riesgo (ecuación 2) fue estimado de manera conjunta usando la metodología de regresiones aparentemente no relacionadas (SUR). El propósito de usar la metodología SUR es identificar de manera consistente el impacto de las variables 
individuales sobre los factores de riesgo, reconociendo que existe una relación estadística entre ellos, y el estimar de forma independiente puede afectar los resultados. Para determinar el ajuste de los modelos se utilizaron criterios de información a nivel individual y global, fijando el nivel de significancia en 10\%. Los cálculos computacionales fueron realizados con STATA 12.0.

\section{RESULTADOS}

En la tabla 1 se observa la estadística descriptiva de las variables individuales. Las variables de autorreporte en general presentan dificultades de medida, tanto por la definición sobre la métrica cualitativa de las mismas, como la dispersión entre las respuestas observadas, por lo que se eliminaron las observaciones extremas que pudieran influenciar el resultado del estudio. En línea con lo encontrado en otras investigaciones, el consumo de alimentos saludables como pescado y fibra es bajo (se declara en promedio consumir entre 1 y 3 veces en el mes), así como la actividad física recreativa (17 minutos/día en promedio), mientras que la percepción de salud y bienestar general promedia entre regular y mala, para el total de la población, siguiendo las categorías usadas en la ENS $(4,16)$.
En cuanto a la relación entre el RCV y los factores de riesgo vinculados con la alimentación, vemos en la tabla 2 una correlación positiva significativa (simple) con todas las variables, siendo la más alta como posible predictor la circunferencia abdominal $(0,36)$ y la glicemia $(0,34)$. Para validar la relación teórica, se realizó una estimación logística de los factores de riesgo sobre el RCV, donde se encontró que, al controlar por edad, sexo, actividad física, consumo de alcohol y tabaco, y factores genéticos, todas las variables de factores de riesgo asociados a la alimentación son significativas (tabla 3). Destaca el impacto del consumo de sal sobre la prevalencia del RCV, lo cual puede explicarse por el efecto indirecto sobre la hipertensión.

La tabla 4 muestra las estimaciones de cada uno de los factores de riesgo sobre las variables individuales, usando la metodología SUR. Se observa que la obesidad abdominal, una vez aplicados los controles, está significativamente relacionada con la percepción física (estado nutricional), y la percepción de salud. En particular, una diferencia en la correcta percepción del estado nutricional puede explicar hasta $6 \mathrm{~cm}$ de circunferencia abdominal. Lo anterior implica que si bien las personas de mayor riesgo están conscientes de tener una peor salud,

\section{TABLA 1}

Variables relacionadas con los factores de riesgo

\begin{tabular}{|c|c|c|}
\hline Variable & Media & $D E^{*}$ \\
\hline Hostilidad=1 & 0,05 & 0,22 \\
\hline Red de apoyo 1 & 3,34 & 1,04 \\
\hline Red de apoyo 2 & 2,59 & 1,19 \\
\hline Red de apoyo 3 & 1,84 & 1,36 \\
\hline Red de apoyo 4 & 3,02 & 1,22 \\
\hline Apoyo económico & 2,03 & 1,42 \\
\hline Estrés financiero & 1,80 & 0,79 \\
\hline Seguridad barrio & 3,99 & 1,90 \\
\hline Diferencia en percepción física & 0,42 & 0,69 \\
\hline Percepción bienestar & 5,43 & 1,18 \\
\hline Percepción salud & 3,28 & 0,89 \\
\hline Actividad física (min/día) & 17,41 & 43,19 \\
\hline Alcohol (días por semana) & 0,57 & 1,36 \\
\hline Diabetes $=1$ & 0,09 & 0,28 \\
\hline Hombre $=1$ & 1,52 & 0,50 \\
\hline$R M=1$ & 0,40 & 0,49 \\
\hline Edad (años) & 41,53 & 17,56 \\
\hline Educación (años) & 10,67 & 3,99 \\
\hline Educación madre (años) & 7,97 & 4,54 \\
\hline Tramo de ingreso del hogar & 5,42 & 2,92 \\
\hline Consumo pescado (días por semana) & 2,84 & 1,01 \\
\hline Consumo fruta/verdura (porciones/día) & 4,60 & 24,46 \\
\hline Consumo cereal integral (días por semana) & 4,94 & 1,54 \\
\hline Hipertensión (antecedente fam.)=1 & 0,34 & 0,55 \\
\hline Colesterol alto (antecedente fam.)=1 & 0,26 & 0,54 \\
\hline
\end{tabular}

*DE: Desviación estándar

Fuente: Elaboración propia en base a ENS 2009. 
tienden a subestimar su estado nutricional. Este efecto es particularmente relevante, ya que si observamos el cuadro 1 , de las personas obesas, sólo $10 \%$ se percibe en dicho estado. Esta información contrasta con lo reportado en el cuadro 2, donde aquellos en estado nutricional de obesidad (efectivo) reportan una peor condición de salud (más del 50\% percibe su situación de salud como regular o mala). Podemos concluir que si bien las personas en situación de riesgo están conscientes de su mala calidad de salud, no ocurre lo mismo con su estado nutricional, lo cual puede explicarse por desinformación o por la incapacidad de analizar dicha información. Estos resultados son extensivos al IMC, donde una percepción incorrecta del estado nutricional explica, en promedio, 3 puntos del índice. Adicionalmente, podemos observar que variables asociadas a la ingesta de alimentos saludables, como un mayor consumo de pescado, y mayor actividad física, tienen un efecto significativo sobre la circunferencia abdominal.

En cuanto al consumo de sal (sodio), existe un efecto significativo del aislamiento social (hostilidad) sobre la calidad de la dieta. Por otra parte, la existencia de apoyo económico de un cercano conduce marginalmente a una dieta más rica en sodio, lo cual puede provenir de un mayor consumo de

TABLA 2

Correlación simple entre factores de riesgo y riesgo cardiovascular.

\begin{tabular}{|c|c|c|c|c|c|}
\hline & RCV & IMC & CA & Colesterol & Glicemia \\
\hline IMC (kgs/mt²) & 0,23 & & & & \\
\hline Circunferencia abdominal (cms.) & 0,36 & 0,86 & & & \\
\hline Colesterol (mg/dL) & 0,24 & 0,24 & 0,29 & & \\
\hline Glicemia (mg/dL) & 0,34 & 0,20 & 0,27 & 0,16 & \\
\hline Consumo de sal (g/día) & 0,17 & 0,29 & 0,35 & 0,08 & 0,05 \\
\hline
\end{tabular}

TABLA 3

Modelo Logit para el riesgo cardiovascular (RCV)

$\begin{array}{lcc} & \text { Coef. } & \text { Valor P } \\ \text { Colesterol (mg/dL) } & 0,009 & 0,000 \\ \text { IMC }\left(\mathrm{kg} / \mathrm{mt}^{2}\right) & 0,012 & 0,000 \\ \text { Obesidad abdominal (cms) } & 0,026 & 0,000 \\ \text { Clicemia (mg/dL) } & 0,008 & 0,000 \\ \text { Consumo sal (gr/día) } & 0,039 & 0,000 \\ \text { Consumo alcohol (días por semana) } & -0,004 & 0,002 \\ \text { Ex fumador =1 } & -0,068 & 0,000 \\ \text { Fumador actual=1 } & 2,526 & 0,000 \\ \text { Fumador ocasional=1 } & -1,463 & 0,000 \\ \text { Diabetes=1 } & 1,086 & 0,000 \\ \text { Edad (años) } & 0,261 & 0,000 \\ \text { Edad } & -0,002 & 0,000 \\ \text { Hombre=1 } & -1,616 & 0,000 \\ \text { Sospecha hipertensión =1 } & 0,849 & 0,000 \\ \text { Antecedente fam. hipertensión }=1 & -0,094 & 0,000 \\ \text { Antecedente fam. Colesterol alto =1 } & 0,147 & 0,000 \\ \text { Actividad física (min/día) } & 0,000 & 0,000 \\ \text { Educación (años) } & -0,058 & 0,000 \\ \text { Escolaridad madre (años) } & 0,053 & 0,000 \\ \text { constante } & -14,604 & 0,000\end{array}$

Nota: Modelo ajustado por criterio de información (Akaike)

Fuente: Elaboración propia en base a ENS 2009. 
alimentos ultra-procesados. Lo mismo se observa de la relación entre el consumo de productos altos en fibra y el nivel de sodio.

En relación al vínculo de las variables individuales sobre el nivel de colesterol total, nuevamente factores sociales, especialmente a nivel de estrés y baja autoestima (en referencia a la red de apoyo), pueden condicionar, en este caso, a la

\section{TABLA 4}

Modelo SUR para factores de riesgo asociados a la alimentación.

\begin{tabular}{|c|c|c|c|c|c|c|c|c|c|c|}
\hline \multirow[t]{2}{*}{ Variable dependiente } & \multicolumn{2}{|c|}{$\begin{array}{l}\text { Obesidad } \\
\text { abdominal }\end{array}$} & \multicolumn{2}{|c|}{$I M C$} & \multicolumn{2}{|c|}{ Colesterol } & \multicolumn{2}{|c|}{ Glicemia } & \multicolumn{2}{|c|}{ Consumo sal } \\
\hline & Coef. & Valor P & Coef. & Valor $\mathrm{P}$ & Coef. & Valor $\mathrm{P}$ & Coef. & Valor P & Coef. & Valor $\mathrm{P}$ \\
\hline Diferencia en percepción física & 5,865 & 0,000 & 3,264 & 0,000 & & & & & & \\
\hline Seguridad ante crimen & & & $-0,132$ & 0,049 & & & & & & \\
\hline Percepción bienestar & $-0,376$ & 0,079 & & & & & 1,276 & 0,041 & & \\
\hline Red de apoyo 1 & & & & & $-2,109$ & 0,032 & & & & \\
\hline Apoyo económico & & & & & $-4,716$ & 0,001 & & & & \\
\hline Diabetes $=1$ & 5,741 & 0,000 & 2,300 & 0,000 & & & 42,75 & 0,000 & & \\
\hline Consumo pescado (porciones/día) & $-0,497$ & 0,006 & & & & & & & & \\
\hline \multicolumn{3}{|l|}{ Consumo cereal integral (días x sem.) } & & & 1,615 & 0,013 & & & 0,094 & 0,026 \\
\hline Hombre $=1$ & $-6,741$ & 0,000 & & & & & $-3,906$ & 0,000 & $-0,884$ & 0,000 \\
\hline Edad (años) & 0,099 & 0,000 & 0,109 & 0,000 & 3,860 & 0,000 & 0,523 & 0,011 & & \\
\hline Edad2 & & & $-0,001$ & 0,000 & $-0,033$ & 0,000 & $-0,004$ & 0,050 & & \\
\hline Educación (años) & & & & & & & & & $-0,039$ & 0,043 \\
\hline Hostilidad=1 & & & & & & & & & 0,788 & 0,003 \\
\hline Actividad física (min/día) & $-0,026$ & 0,007 & $-0,008$ & 0,061 & & & & & & \\
\hline Antecedente fam. Colesterol alto $=$ & $1-2,238$ & 0,000 & & & $-4,816$ & 0,013 & & & & \\
\hline Antecedente fam. hipertensión $=1$ & & & $-0,984$ & 0,000 & & & & & & \\
\hline Escolaridad madre (años) & $-0,237$ & 0,001 & $-0,052$ & 0,081 & & & & & $-0,056$ & 0,001 \\
\hline Constante & 103,8 & 0,000 & 26,55 & 0,000 & 111,5 & 0,000 & 76,72 & 0,000 & 11,57 & 0,000 \\
\hline
\end{tabular}

\section{CUADRO 1}

Auto-percepción del estado nutricional para el grupo en situación de obesidad (\%).

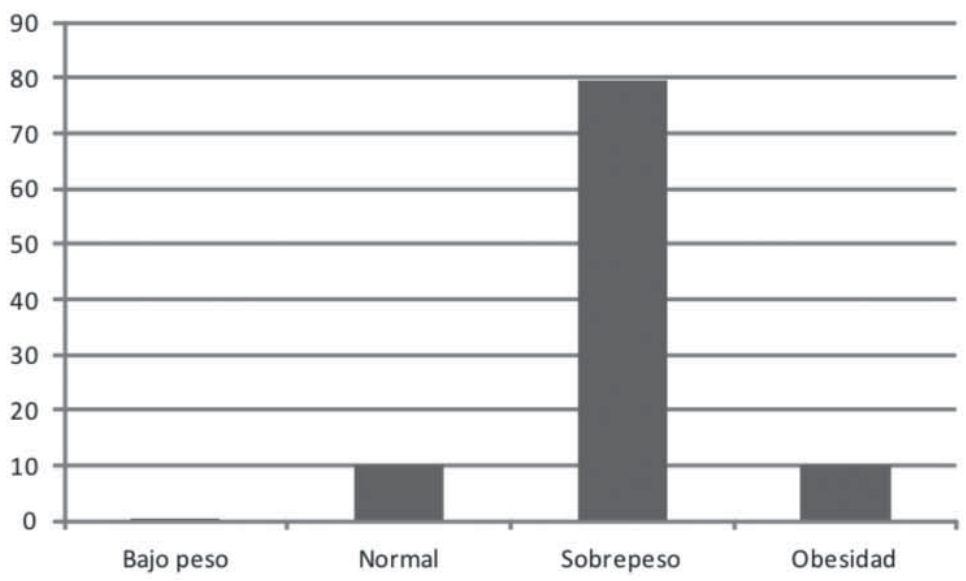


incapacidad de llevar una dieta saludable, lo cual puede argumentarse tanto por acceso económico, como por autocontrol en la ingesta de productos altos en colesterol. Adicionalmente destaca el impacto que tiene el menor consumo de fibra con el incremento en el colesterol total. Finalmente, todo lo demás constante, el nivel de glicemia total se encuentra relacionado tanto con aspectos individuales, como la percepción de salud, así como el entorno, en este caso visto como la existencia de apoyo económico, sin embargo este último efecto es de menor orden de magnitud.

\section{DISCUSIÓN}

A pesar de las sustanciales mejoras en la calidad de vida y acceso a la salud en Chile durante los últimos años, la prevalencia de los factores de riesgo a la salud vinculados a la nutrición se ha incrementado o mantenido en el tiempo, particularmente en los grupos más jóvenes (15). Reducir los factores de riesgo (y promover los factores protectores de la salud) es una de las líneas de trabajo prioritarias del Ministerio de Salud, según lo expuesto en la Estrategia Nacional 2011-2020. Sin embargo hay escasa investigación sobre la relación entre variables individuales (conductuales, ambientales, sociales) y prevalencia de los factores de riesgo (4). En particular, en cuanto a los factores conductuales y sociales, no existe evidencia para Chile respecto del impacto que ellos tienen sobre la prevalencia de factores de riesgo asociados a la alimentación.

En este estudio analiza estadísticamente los determinantes de los factores de riesgo asociados a la salud nutricional, usando variables individuales provenientes de la Encuesta Nacional de Salud 2009-2010, mediante una estimación de ecuaciones aparentemente no relacionadas (SUR). A partir de los resultados se encontró que, controlando por variables económicas, demográficas y genéticas, existen determinantes sociales y conductuales que explican significativamente el incremento (o disminución) de la prevalencia de los factores de riesgo. En línea con la literatura empírica, los factores de riesgo aumentan con la edad de forma cóncava y disminuyen en la medida que aumenta el nivel de socioeconómico (medido indirectamente por la escolaridad de la madre) (8-10). Asimismo, los factores genéticos de prevalencia de enfermedades como el género de la persona son determinantes en los factores de riesgo $(3,11)$. Adicionalmente, se aprecia que existe una relación directa entre el mayor consumo de productos saludables (pescado y cereales altos en fibra) y la reducción de la prevalencia de los factores de riesgo. Es cuanto a los factores conductuales, es de particular relevancia el efecto de la percepción que la persona tiene sobre su salud, condición física e identificación con el entorno. Además, se encuentra que la existencia de una red de apoyo a nivel social y económico a nivel individual es también relevante para reducir la prevalencia de los factores de riesgo observados. Estos resultados están en línea con lo encontrado en otros estudios (7 - 9).

El efecto que tiene la discrepancia entre el estado nutricional percibido (autorreporte) y el efectivo (medición) es particularmente relevante, ya que indica que una subestimación en la condición de obesidad del sujeto conduce al incremento de su condición de riesgo (medido como mayor IMC y circunferencia abdominal). Lo anterior es preocupante, ya que en el grupo de obesidad en particular, sólo uno de cada diez reconoce su estado nutricional efectivo. Evidencia internacional en estudios para adolescentes y adultos muestra que el sesgo en la percepción del estado nutricional incide en la subestimación del riesgo de salud $(17,18)$ y que dicha subestimación está vinculada con la carencia de capital humano (18 - 21).

En suma, los resultados muestran que si bien la población chilena aún enfrenta dificultades de acceso (particularmente económico) a una dieta nutritiva y saludable (22), el conocimiento de las personas respecto de su situación nutricional efectiva y la capacidad de generar y mantener redes de apoyo son herramientas de alto impacto al momento de diseñar intervenciones de política pública alimentaria que conduzcan a alcanzar la Seguridad Alimentaria y Nutricional. Esto sugiere tener particularmente en cuenta aquellos programas que tienen la oportunidad de vincular estrategias de educación alimentaria y participación local inclusiva, como los programas de asistencia directa (PAE y PNAC), así como los programas de

\section{CUADRO 2}

Auto-percepción de salud según estado nutricional (\%).

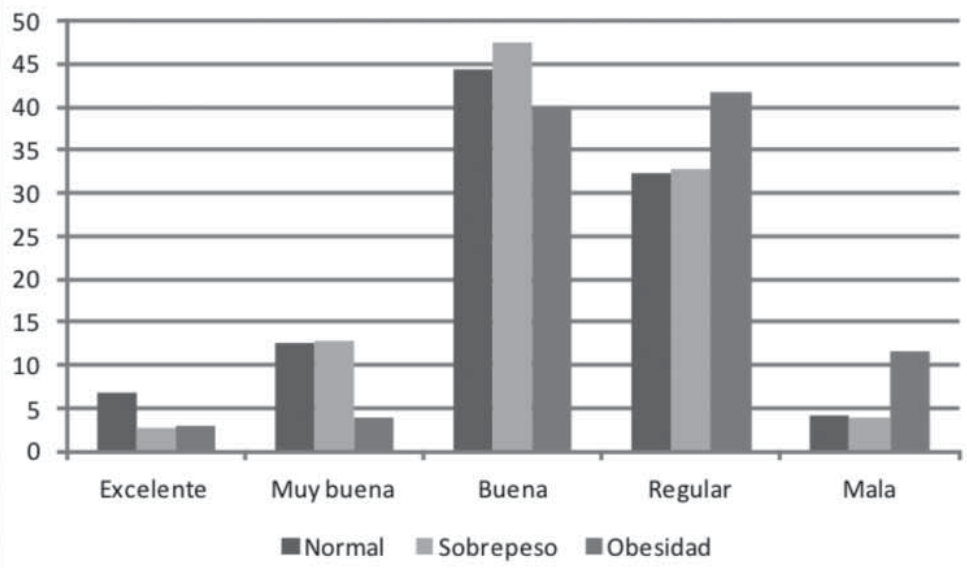


educación en escuelas públicas. La vinculación de todos los actores del proceso (padres, hijos, comunidad), mediante la articulación a través de profesionales del área alimentación, es clave para el éxito de los programas, lo cual demanda mayor capacitación e incorporación de nutricionistas, cocineros y otros profesionales $(15,16)$. Adicionalmente, es necesario reconocer la heterogeneidad intra e intergrupal en el acceso y comprensión de la información nutricional, particularmente por nivel socioeconómico, como elemento clave en el diseño de políticas al consumidor, tanto de información como de educación alimentaria. Lo anterior implica reconocer el entorno social y la oferta alimentaria disponible en cada grupo de referencia (23).

\section{RESUMEN}

Los factores de riesgo a la salud asociados con la alimentación son un foco clave en las estrategias de promoción de la salud y de seguridad alimentaria y nutricional en Chile (SAN). Este artículo entrega un análisis de los efectos que variables conductuales y sociales tienen sobre los factores de riesgo asociados a una mala nutrición (obesidad, altos niveles de colesterol, glicemia elevada y altos niveles de sodio), en base a la Encuesta Nacional de Salud 2009-2010 utilizando una estimación por regresiones aparentemente no relacionadas (SUR). Los resultados revelan que las variables asociadas a aspectos conductuales y sociales tienen un impacto significativo en los factores de riesgo de salud alimentaria, controlando por variables demográficas, genéticas y socioeconómicas. Destaca el efecto de la subestimación de las personas respecto a su estado nutricional, que puede explicar hasta $6 \mathrm{~cm}$ de circunferencia abdominal y 3 puntos en el IMC. Asimismo, las redes de apoyo social y económico a nivel individual juegan un rol fundamental. Los resultados entregados son un insumo relevante para la discusión sobre los mecanismos que la política pública debiese tener a fin de promover la seguridad alimentaria y nutricional, en particular al diseñar programas de educación e información a la población, donde existe la posibilidad de fortalecer las redes de apoyo existentes.

Palabras clave: riesgo de salud, estado nutricional, seguridad alimentaria nutricional, Chile, malnutrición.

Declaración de conflicto de interés: Los autores declaran que no tienen conflicto de interés de ningún tipo, ni real o potencial sobre los resultados presentados.

Agradecimientos: Expreso mis agradecimientos a Fernando Vio y Pablo Faret por su significativa contribución a este estudio, a través de sus comentarios y apoyo durante la investigación.

\section{BIBLIOGRAFÍA}

1. Organización Mundial de Salud: Diet, nutrition and the prevention of chronic diseases. WHO Technical Report Series 916, 2003.

2. Popkin, B: Global nutrition dynamics: the world is shifting rapidly toward a diet linked with non-communicable diseases. Am J Clin Nutr. 2006:84(2):289-98.

3. Yusuf, S; Ounpuu, S; Bautista, L; Franzosi, G; Commerford P; Lang, C et al.: Obesity and the risk of myocardial infarction in 27000 participants from 52 countries: a case-control study. Lancet 2005; 366:1640-49.

4. Ministerio de Salud: Estrategia Nacional de Salud 2011-
2020. MINSAL, 2011.

5. Albala, C; Vio, F; Kain, J; Uauy, R: The nutrition transition in Chile. Public Health Nutr 2002;5 (1A):123-8.

6. Muzzo, S. y Mockenberg, B. Reflexiones sobre el aumento de la obesidad en Chile. Rev Chil Nutr 2012; 39 (4), p: 113-5.

7. Anderson, E; Winett, R; Wojcik, J: Self-regulation, selfefficacy, outcome expectations, and social support: Social cognitive theory and nutrition behavior. Ann Behav Med. 2007; 34, Issue 3. (3):304-12.

8. Roos, E; Lahelma, E; Virtanen, M; Prättälä; Pietinen P: Gender, socioeconomic status and family status as determinants of food behaviour. Social Sci Med. 1998, 46, Issue 12. (12):1519-29.

9. Shepherd, Richard: Social determinants of food choice. Proc Nutr Soc. 1999;58, Issue 4, (4):807-12.

10. Groth, MV; Fagt, S; Brondsted, L: Social determinants of dietary habits in Denmark. European J Clin Nutr. 2001. Nov;55(11):959-66.

11. Margozzini P. ¿Cuál es la definición de obesidad que mejor predice el riesgo cardiovascular? Comentario Editorial. Rev Chil Cardiol. 2006. 25(2):185-9.

12. Williams, G; Minicucci, D; Kouides, R; Levesque, C; Chirkow, V; Ryan, R; Deci, E: Self-determination, Smoking and Health. Health Education Res. 2002. 17 (5):512-21.

13. Wilson, $P$; D'Agostino, R; Sullivan, L; Parise, $H$; Kannel, W: Overweight and obesity as determinants of cardiovascular risk. Arch Intern Med. 2002; 162(16):1867-72.

14. Ministerio de Salud, Informe Encuesta Nacional de Salud 2009-2010. MINSAL, 2010.

15. Salinas, J. y Vio, F: Programas de salud y nutrición sin política de Estado: el caso de la promoción de la salud escolar en Chile. Rev Chil Nutr. 2011; 38 (2), 100-6.

16. Vio, F., Salinas, J., Lera L., González, CG., Huenchupán, $M$ : Conocimientos y consumo alimentario en escolares, sus padres y profesores: un análisis comparativo. Rev Chil Nutr. 2012; 39 (3), 34-9.

17. Brener, ND., Eaton, D., Lowry, R., McManus, T: The Association between Weight Perception and BMI among High School Students. Obesity. 2004. 12(11): 1866-74.

18. Yannakoulia, M., Panagiotakos, DB, Pistavos, C, Stefanadis C: Correlates of BMI Misreporting among Apparently Healthy Individuals: The ATTICA Study. Obesity, 2006. 14(5):894-901.

19. García-Mendizabal, M., Carrasco, JM, Pérez-Gomez, B, et al: Role of educational level in the relationship between Body Mass Index (BMI) and health-related quality of life (HRQL) among rural Spanish women. BMC Public Health 2009. Apr 30;9:120.

20. Metcalf PA, Scragg RK, Willoughby P, Finau S, TipeneLeach D. et al: Ethnic differences in perceptions of body size in middle-aged European, Maori and Pacific people living in New Zealand. Internat $j$ Obes Related Metabolic Dis. 2000,;24(5):593-9.

21. Nawaz, H; Chan, W; Abdulrahman, M; Larson, D; Katz, D: Self-reported weight and height: Implications for obesity research. Am J Preventive Medic 2001; 20 (4), Issue 4, 294-8.

22. Ministerio de Salud. Resultados de la Encuesta Nacional de Consumo Alimentario 2010-2011. MINSAL 2014.

23. Viswanath, $K$; Bond, $K$ : Social Determinants and Nutrition: Reflections on the Role of Communication. J Nutr Educ Behav. 2007:39(2 Suppl):S20-4. 